\title{
Monitoring Development Sustainability through Sustainable Community Indicators ${ }^{+}$
}

\author{
Somchai Jitsuchon \\ Thailand Development Research Institute, Bangkok 10310, Thailand; sj.tdri@gmail.com \\ + Presented at the Economy, Sustainable Development and Energy International Conference (ESDEIC), \\ Edinburgh, Scotland, UK, 25-27 June 2018.
}

Published: 26 October 2018

\begin{abstract}
The Sustainable Development Goals (SDGs) is both a continuation to the Millennium Development Goals (MDGs) and an improvement on the addition of 'means of implementation' to achieve the goals. The SDGs recognize that countries should have their own ways to achieve development goals. In this regard, Thailand, through its 'sufficiency economy philosophy' (SEP) invented by the late King Bhumibol Adulyadej, has created a unique path to attain sustainable development. The SEP path involves (a) shaping personal attitudes and behaviors towards sufficiency and (b) setting procedures or protocols for development projects and programmes. In this paper, we developed a system of indicators that capture the first component-personal attitudes and behaviors - of the sufficiency economy philosophy practiced by Thai people, as well as a set of ultimate development outcome indicators, using data from nation-wide household and community surveys. We then analyze how practicing the sufficiency economy philosophy is associated with development outcomes, where we find positive correlation between the two groups of indicators.
\end{abstract}

Keywords: sustainable development indicators; monitoring and evaluation; King Bhumibol Adulyadej; sufficiency economy philosophy

\section{Introduction}

The Sustainable Development Goals (SDGs) is both a continuation to the Millennium Development Goals (MDGs) and an improvement on the addition of 'means of implementation' to achieve those goals. SDGs recognize that countries should have their own ways to achieve development goals. In this regard, Thailand, through its well-known 'sufficiency economy philosophy' (SEP), invented by the late King Bhumibol Adulyadej of Thailand, created a unique path to attain sustainable development. The SEP path involves (a) shaping personal attitudes and behaviors towards sufficiency and (b) setting procedures or protocols for development projects and programmes. In this paper, we developed a system of indicators that captures the first componentpersonal attitudes and behaviors - of the sufficiency economy philosophy, as practiced by Thai people, as well as a set of ultimate development outcome indicators, using data from a well-designed, nation-wide household survey. The resulting indicators can be used to monitor how closely Thai people are practicing the SEP principles. More importantly, an analysis of the correlation will demonstrate how practicing the SEP can lead to better development outcomes.

\section{The Sufficiency Economy Philosophy}

As a scientific-minded Buddhist, King Bhumibol Adulyadej developed SEP to be, as described in Avery and Bergsteiner [1] (p. xvii), "a virtues- and science-based decision making framework or foundation that can be applied to all spheres of life". Figure 1 summarizes the conceptual structure of SEP, 
the so-called ' 3 loops, 2 conditions' diagram. The three loops consist of moderation, reasonableness and resilience, which are meant to be practiced by those following the SEP. The practices must be based on two conditions: knowing how to do it (knowledge) and acting with high moral standards (virtue).

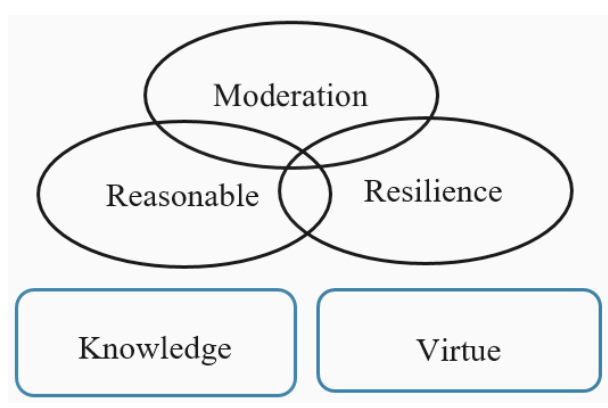

Figure 1. Sufficiency Economy Philosophy Concept Diagram.

There are many more details involved in each of these five keywords. Moderation derives its root from Buddhism's 'middle way' thinking, focusing on how one should moderate one's need, not to be too greedy, especially without consideration of resilience. For example, excessive consumption beyond one's means would cause over-indebtedness. Being reasonable has many interpretations and numerous applications. Taking consumption, reasonable consumption would mean purchasing goods and services that one really needs, not driven by jealousy of others. Resilience means being able to cope with negative shocks, through both ex-ante and ex-pose risk management.

The two conditions, knowledge and virtue, are just as important. The SEP emphasizes use of knowledge when living life, and in designing and implementing development projects. The King often stressed that 'knowledge' must be used properly and adapted to local and project context. For example, he always experimented and adapted 'new' agricultural techniques, but only disseminated them when the results of experiments were satisfactory. The second condition of virtue is quite unique and places importance on the inner virtue of people. Virtues may include honesty, perseverance, sharing, loving, self-reliance, and public mindedness. The SEP regards these qualities as necessary for sustainability. The SEP also shares the international concept of sustainability regarding equality and natural environment. By continually raising the poorest Thai's quality of life, reduction of inequality will entail. King Bhumibol's teachings of living in harmony with nature is proof of the SEP's high regard of sustainable environment practices.

\section{Methodology of Constructing Sustainable Community Indicators (SCI)}

See Thailand Development Research Institute [2] for more details on how SCI is constructed. The Sustainable Community Indicators (SCI) is constructed scientifically, using information collected following accepted statistical sampling methods and are able to capture whether Thai people are practicing SEP in a natural way, meaning that indicators are derived from peoples' normal lifestyle or normal Thai ways of living. We define behaviors that follow the SEP principle to be comprised of 15 elements: moderation, resilience, prudence, knowledge, perseverance, honesty, sharing, reasonableness, selfreliance, public minded, innovation, social coherence, explosion-from-within, leadership, and environmental protection. We define development outcomes as comprising of 12 elements: happiness, sufficient consumption, sufficient income, poverty-free, education, health, employment, decent house, asset, equality, social capital and a good natural environment. Consequently, SCI can be broken down to two major components: one capturing SEP behavior, called SCI-SEP and another one capturing development outcomes, called SCI-OC.

Each element of SCI_SEP and SCI_OC was derived from a set of questions in the questionnaire. For SEP behaviors and thinking the questions are based on facts, behaviors, thought processes, opinions about facts and behaviors of other members in the community and opinions about development projects in the community. The settings of all questions were related to day-to-day behaviors, normal life decisions or situations most Thais are familiar with, using simple wording in 
Thai language. For example: how well are you satisfied with your life? Do you plan your spending monthly? Does the family seek additional income sources to diversify income risk? Do you try to control expenses and how? How do you seek knowledge that will help you earn more income? Will valuable things be lost if your house door is left open or property is left unattended in this community? How would a typical member' of the community choose a public figure between an honest but ineffective and an effective but dishonest one? Do you use organic fertilizer, and why?

SCI-SEP and SCI-OC are normalized to have values between 0 and 100: with 100 given for following SEP fully or having the best development outcomes. The scaling is subjective but intuitive. For example, for the question 'how closely do members of this community feel towards each other?' an answer of 'very distantly' gets a 0 score, 'distantly' gets 25 , 'neither distantly nor closely' gets 50 , 'closely' gets 75, and 'very closely' gets 100. The SCI-SEP and SCI-OC are simple averages of the element scores. They can be displayed both at aggregate and at element level. Furthermore, they can also be shown geographically at national level, regional level and provincial level. This is useful in the monitoring of progress in regards to how well Thai people practice the SEP.

\section{Results}

\subsection{SCI Scores}

Table 1 and 2 show the SCI-SEP and SCI-OC scores, as well as their elements' scores, for the Northeast region (non-municipality only) for 2017. SCI-SEP scored 64 with the highest element score of 85.5 for public mindedness, followed by explosion-within and reasonableness. Knowledge seeking received the lowest score, meaning the Northeasterners were not very active in seeking knowledge. For SCI-OC, the aggregate score is 60.6 with the highest element scores in health, environment status and decent houses. The lowest outcomes are sufficient consumption and income, which is consistent with the fact that the Northeast is the poorest region of Thailand.

Table 1. SCI-SEP and its element scores, Non-municipal Northeast 2017.

\begin{tabular}{ccc}
\hline SEP Elements & Mean & Standard Deviation \\
\hline SCI-SEP & 64.1 & 9.8 \\
moderation & 61.7 & 15.7 \\
resilience & 52.4 & 19.2 \\
prudence & 61.4 & 22.5 \\
knowledge seeking & 42.2 & 19.4 \\
perseverance & 70.5 & 28.5 \\
honesty & 53.6 & 20.1 \\
sharing & 63.1 & 23.0 \\
reasonableness & 77.0 & 21.7 \\
self-reliance & 70.0 & 16.6 \\
public-minded & 85.6 & 17.8 \\
Innovation & 64.6 & 42.0 \\
social coherence & 70.0 & 25.0 \\
explosion-from-within & 78.6 & 15.4 \\
leadership & 56.7 & 17.4 \\
environmental protection & 54.4 & 24.7 \\
\hline
\end{tabular}


Table 2. SCI-OC and its element scores, Non-municipal Northeast 2017.

\begin{tabular}{ccc}
\hline Outcomes & Mean & Standard Deviation \\
\hline SCI-OC & 60.6 & 8.3 \\
happiness & 70.0 & 14.2 \\
sufficient consumption & 11.3 & 19.2 \\
sufficient income & 33.4 & 30.3 \\
poverty-free & 70.0 & 19.4 \\
education & 74.0 & 11.1 \\
health & 87.9 & 7.4 \\
employment & 58.2 & 25.9 \\
decent house & 77.9 & 5.6 \\
asset & 64.3 & 16.0 \\
community equality & 62.4 & 16.8 \\
social capital & 36.2 & 23.4 \\
environmental status & 81.9 & 12.4 \\
\hline
\end{tabular}

\subsection{Correlation between SCI-SEP and SCI-OC}

We have postulated before that SEP can offer a path to sustainable development. An analysis of correlation and causality is needed to test this hypothesis. We calculated simple correlation coefficients (a) between SCI-SEP and SCI-OC (b) between elements of SCI-SEP and SCI-OC (c) between SCI-SEP and elements of SCI-OC and (d) between elements of SCI-SEP and elements of SCIOC. Firstly, we found a positive correlation of 0.345 between SCI-SEP and SCI-OC (with significance level of less than 1\%). Secondly, we found that SCI-SEP is positively correlated (again significantly) with all SCI-OC elements except environmental status. In addition, SCI-OC is significantly positively correlated with all SCI-SEP elements except moderation.

For elements-to-elements correlation, we found significantly positive correlations in 149 pairs (out of 180 possible pairs), 30 negative correlating pairs and 1 no-correlation pair. Thus, SCI-SEP and SCI-OC are mostly moving in the same direction, as shown in Figures 2 and 3.

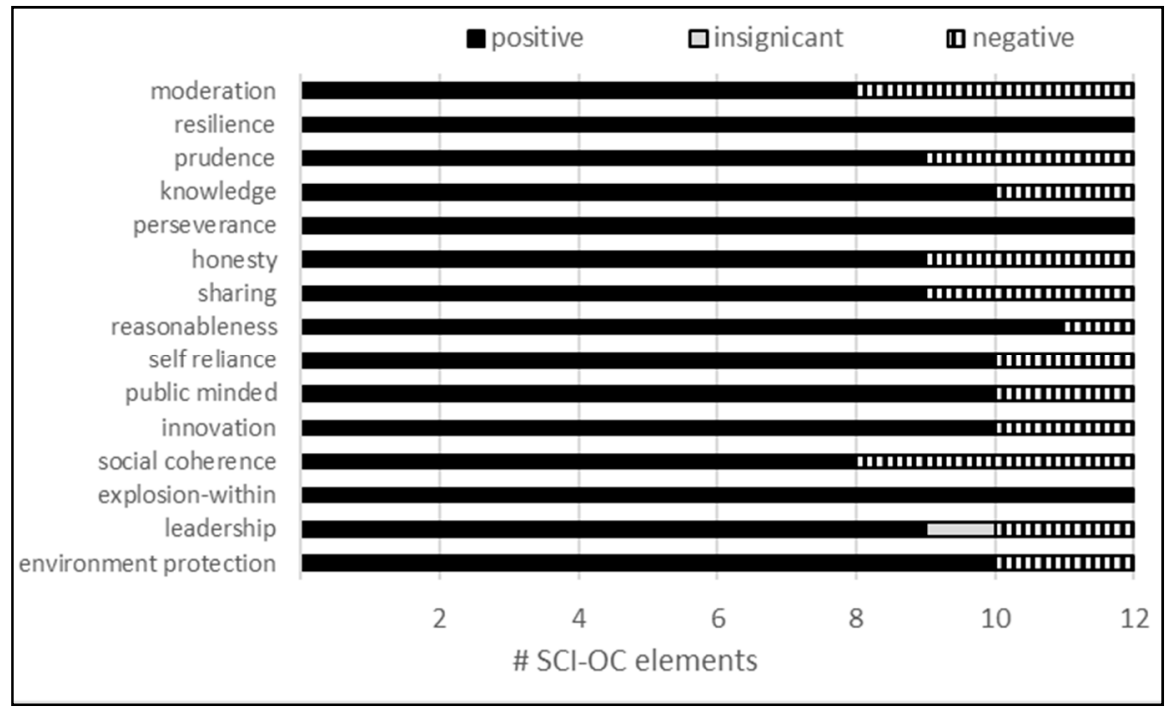

Figure 2. Correlation coefficients between SCI-SEP element and development outcomes. 


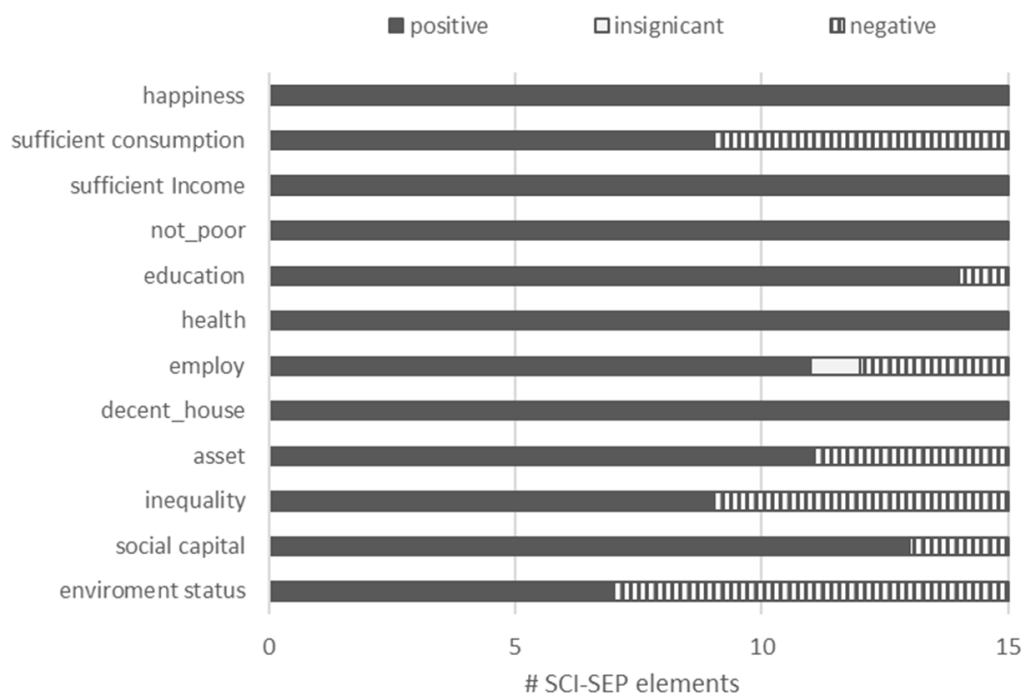

Figure 3. Correlation coefficients between each development outcome and SEP elements.

Figure 2 shows that all SEP elements, except social coherence, have a positive correlation with at least 9 out of 12 SCI-OC development outcomes. Resilience, perseverance and explosion-fromwithin are correlated positively with all development outcomes, suggesting their strong contribution to better outcomes. Figure 3 shows that happiness, sufficient income, health, and decent house are positively correlated with all 15 SEP elements, suggesting that practicing SEP 'wholly' is likely leading to these outcomes.

\section{Conclusions and Applications of SCI}

This paper reports the construction of 'sustainable community indicators' (SCI), which consists of a set of indicators capturing the practice of SEP (SCI-SEP) and a set of indicators highlighting development outcomes (SCI-OC), using data from national household surveys. The initial findings from the Northeastern region dataset is promising, and shows that strong positive correlations exist between SCI-SEP and SCI-OC. SEP may then be said to offer a possible 'path' to sustainable development. To secure such claims, a time series of SCI needs to be constructed and further analysis of whether SEP's contribution to better development outcomes is robust over time needs to be done. We plan to do regression analysis for this purpose. Another application of SCI is as a tool to evaluate area-based development projects where a double-difference analysis can be performed on datasets collected for SCI.

\section{References}

1. Avery, G.C.; Bergsteiner, H. Editors' Preface. In Sufficiency Thinking: Thailand's Gift to an Unsustainable World; Avery, G.C., Bergsteiner, H., Eds.; Allen \& Unwin: New South Wales, Australia, 2016; pp. xiv-xviii.

2. Thailand Development Research Institute. A Construction of Sustainable Community Indicators. Unpublished report submitted to the Thailand Sustainable Development Foundation, 2018.

(C) 2018 by the author. Licensee MDPI, Basel, Switzerland. This article is an open access article distributed under the terms and conditions of the Creative Commons Attribution (CC BY) license (http://creativecommons.org/licenses/by/4.0/). 